\title{
Laboratory Modeling of the Downwelling Coastal Current and the Related Bottom Ekman Layer above the Inclined Bottom in the Rotating Homogeneous and Stratified Fluid
}

\author{
D. N. Elkin*, A. G. Zatsepin \\ Shirshov Institute of Oceanology, Russian Academy of Sciences, Moscow, Russian Federation \\ *e-mail: dmelkin@mail.ru
}

\begin{abstract}
Laboratory experiments on studying the downwelling coastal current above the inclined bottom are conducted in the tank placed at a rotating platform. The current is formed by a source of constant flow of water with the same density (barotropic case) or the lower one (baroclinic case) as compared to the water in the tank. It is revealed that both in the barotropic and the baroclinic (under certain conditions) cases, the bottom Ekman layer formation implies sinking of the downwelling coastal current water. Some regularities in dynamics of the downwelling coastal current and the associated bottom Ekman layer in the barotropic and baroclinic cases are determined. It is found that in the barotropic case, the water in the bottom Ekman layer spreads from the source down the slope. It is shown that in the baroclinic case when the densities of water in the source and in the tank do not differ significantly, the water with lower density from the source enters the bottom Ekman layer which undergoes convective instability. The criteria for transition from the barotropic current regime to the baroclinic one, i. e. to the convective instability of the bottom Ekman layer are determined. It is revealed that convection in the bottom layer develops at the Rayleigh number values considerably exceeding its critical values characteristic of the laminar boundary layer in a rotating fluid. Actually, the bottom Ekman layer in the experiments is not purely laminar; its thickness is much higher than that of the laminar flow. Consequently, the coefficient of effective viscosity in this layer is also higher than the kinematic viscosity value which is used for calculating the critical Rayleigh number. When the densities of water in the source and in the tank differ significantly, the water with lower density propagates near the surface, does not enter the bottom Ekman layer and does not undergo convective instability.
\end{abstract}

Keywords: downwelling coastal currents, laboratory modeling, inclined bottom, water stratification, rotating platform, bottom Ekman layer, convective instability, mixing.

Acknowledgements. The present study was carried out under the framework of the State Order No. 0149-2018-0003. It is supported by RSF Grant No. 14-50-00095 concerning the analysis of the results and by RFBR Grant No. 17-05-00381 - concerning the article preparation for the publication.

For citation: Elkin, D.N. and Zatsepin, A.G., 2018. Laboratory Modeling of the Downwelling Coastal Current and the Related Bottom Ekman Layer above the Inclined Bottom in the Rotating Homogeneous and Stratified Fluid. Physical Oceanography, [e-journal] 25(3), pp. 165-176. doi:10.22449/1573-160X-2018-3-165-176

DOI: $10.22449 / 1573-160 X-2018-3-165-176$

(C) 2018, D. N. Elkin, A. G. Zatsepin

(C) Physical Oceanography

\section{Introduction}

The ocean and sea ventilation mechanisms have been an actual object of research, at least since the issue of the famous W. Munk article concerning this topic [1]. One of such mechanisms is the water sinking in the bottom Ekman layer on the shelf/continental slope and their convective mixing with overlying stratified waters [2]. In the presence of a coastal current reaching the bottom, the bottom Ekman layer is formed. The integral water transfer in this layer is perpendicular to the direction of current and to the left of it (in the Northern Hemisphere). It is obvious 
that in the bottom Ekman layer there is a transfer of water from the coast, accompanied by its descent along an inclined bottom in cases of either a cyclonic current along the coast or anticyclonic circulation around an island. This type of current is called downwelling, in contrast to upwelling, having the opposite direction, in bottom Ekman layer where the rise of water occurs [3].

Stable density stratification of the aquatic environment restricts the depth of descent or rising of water in the bottom Ekman layer [4]. Regarding the downwelling current, reaching a certain depth of immersion, the water in the bottom Ekman layer, as more light in relation to the overlying waters, may undergo convective instability and mix with them [5]. The mixing products can be introduced into the surrounding stratified water column in the form of quasi-isopycnic intrusions.

In view of the widespread prevalence of coastal downwelling currents in the seas and oceans, this process should occur very often and play a certain role in the stratified water column ventilation. In [6] it was suggested to be one of the important factors of oxygen ventilation of the active layer of the Black Sea and the retention of the upper boundary of the hydrogen sulphide zone in the permanent pycnochaloklin area. Despite some evidence of the implementation of this process, there is currently no reliable evidence of its implementation in the Black Sea and other seas. To a certain extent, this is due to its sporadic nature, its closeness to the bottom and its small scale. Therefore, the methods of laboratory and numerical modeling can be of particular importance in studying of this problem.

In [7], the coastal downwelling current and the bottom Ekman layer (with the sinking of waters on an inclined bottom) formed by it, were simulated in the laboratory. The experiments were carried out both in a homogeneous and in a two-layered stratified aquatic environment. In the second case, the phenomenon of less dense water sinking in the bottom Ekman layer and the convective instability of this layer were detected. However, due to the limited number of experiments, the regularities of the dynamics of the coastal downwelling current and the bottom Ekman layer associated with it have been studied quite approximately. Preliminary estimates of the bottom Ekman layer parameters on the continental shelf/slope of the Black Sea were made based on the phenomenological results of the laboratory experiment, as well as on the results of in situ studies of the near-bottom boundary layers and theoretical representations [7]. In addition, the possible depth of less dense water sinking in this layer, reaching $50 \mathrm{~m}$, was calculated.

The present paper was aimed to continue the laboratory experiments started in [7], for a more detailed study of the dynamics of coastal downwelling flow of water with the same or lower density as compared to the water in the tank, as well as the bottom Ekman layer formed on this inclined bottom formed by this current. As a result of this research, certain theoretical concepts were tested for a homogeneous density of the aquatic environment, and some constants were established to quantify the parameters of the coastal current and bottom Ekman layer. For a two-layer stratified current, the critical values of parameters at which the less dense water sinking in the bottom Ekman layer and its convective instability development is possible were found. 


\section{Laboratory plant}

The experiments were carried out on the laboratory plant (Fig. 1). In the center of the rotating platform (1) with a diameter of $1 \mathrm{~m}$ with the possibility of a smooth change of its rotation speed, a cylindrical tank of plexiglas (2) with a radius of $30 \mathrm{~cm}$ and a height of $27 \mathrm{~cm}$ is filled with a $\mathrm{NaCl}$ aqueous solution to a certain level. At the bottom of the tank, there is a cone (3) inscribed in it, made of thin glasscloth $(0.3 \mathrm{~mm})$. The inclination angle of the conical surface to the horizontal is $\alpha=$ $=30^{\circ}$. The cone height is $14 \mathrm{~cm}$. At the top of the cone there is a vertical hole $10 \mathrm{~mm}$ in diameter, connected by a hose with the Mariotte bottle (4). A hollow cylinder with a lid $16 \mathrm{~cm}$ in diameter and $2 \mathrm{~cm}$ in height, sealed on the cone surface, is axially symmetric above this hole. In the upper part of the cylinder, under the cover, there is a gap $0.5 \mathrm{~cm}$ wide - a ring source (5). Through this source, during the experiment, from the Mariotte bottle with a steady flow a tinted water of a given density - either the same as the water in the tank, or less. Under the influence of the Coriolis force, the water entering the tank forms an anticyclonic frontal current, the form of which is recorded from above with the help of a video camera fixed on the tank top (6) and from the side - by the side video camera (7). The speed of this flow is determined by automatically measuring the movement of round paper pellets with a diameter of $1 \mathrm{~mm}$ floating on the water surface.

Rate of the ring source varied from experiment to experiment within the range of values $Q=4-10 \mathrm{~cm}^{3} / \mathrm{s}$. The rate value was set before each experiment and was kept constant to within $5 \%$ with the help of the Mariotte bottle.

As already noted above, two series of experiments were carried out. In the first series, the salinity (density) of water in the source was approximately the same as in the tank: $\rho_{1} \approx \rho_{0}$, where $\rho_{1}$ is the density of water in the source, and $\rho_{0}$ is the water density in the tank (barotropic case). In the second series, the water in the source had a lower density than the water in the tank: $\rho_{1}<\rho_{0}$ (baroclinic case). The water temperature in the source and in the tank coincided with an accuracy of $\pm 0.3^{\circ} \mathrm{C}$
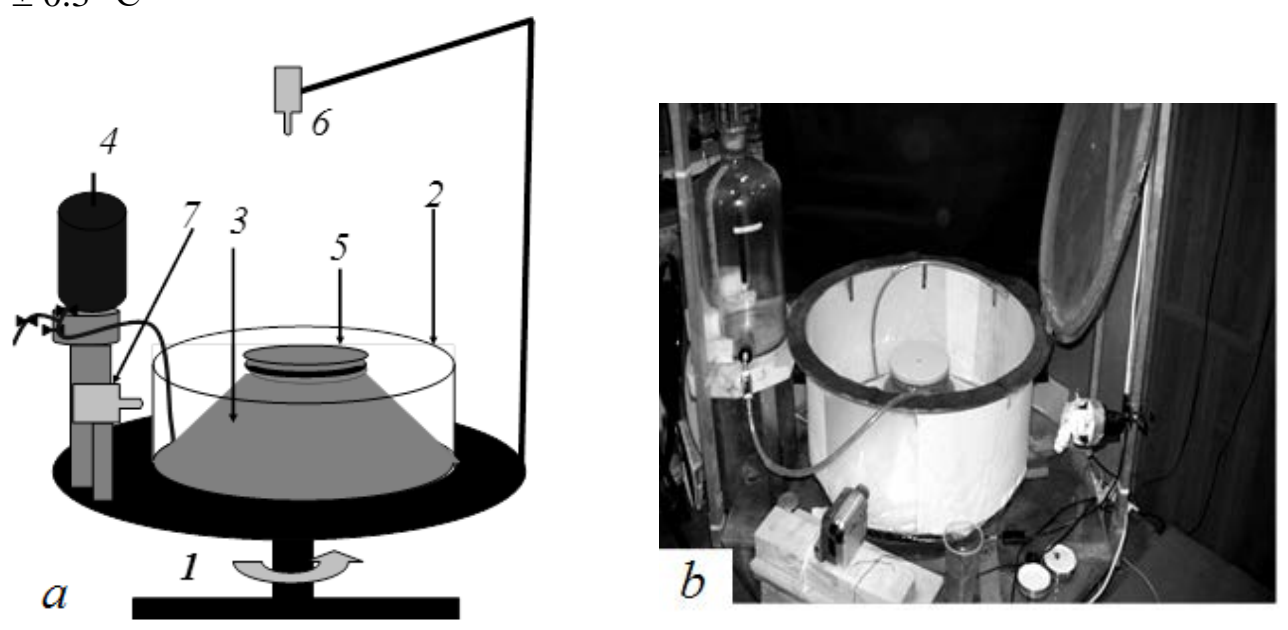

Fig. 1. Scheme and photograph of the laboratory plant: $a$-plant scheme: 1 is a rotating platform, 2 is the tank, 3 is the truncated cone, 4 is the Mariotte bottle, 5 is the slot source of mass and buoyancy, 6 is the video camera, 7 is the lateral/side video camera; $b$ - photograph of the plant

PHYSICAL OCEANOGRAPHY VOL. 25 ISS. 3 (2018) 
In the experiments of the first series, the water in the tank and in the source was fresh and distilled. In the experiments of the second series, the water in the tank was salty, with salinity varying from experiment to experiment, while in the source it was fresh. As one of the basic dimensional parameters, the reduced free fall acceleration $g^{\prime}=g \Delta \rho / \rho$, where $g$ is the free fall acceleration, $\Delta \rho=\rho_{1}-\rho_{0}$ is the density difference of the water in the source (the Mariotte bottle) and in the tank. At that, the accuracy of $g^{\prime}$ definition was $\pm 0.1 \mathrm{~cm} / \mathrm{s}^{2}$. Other important dimensional parameters of the experiment are the volume rate of the source $Q$ (the accuracy of its definition is $\pm 0.5 \mathrm{~cm}^{3} / \mathrm{s}$ ) and the Coriolis parameter $f=2 \Omega$, where $\Omega=$ $=2 \pi / T$ - is the angular rotation speed of the platform, and $T$ is the its rotation period (accuracy of its definition is not lower than $0.5 \%$ ). The kinematic viscosity of the fluid, also included in the number of important dimensional parameters, was approximately constant and equal to $10^{-2} \mathrm{~cm}^{2} / \mathrm{s}$.

\section{Results of the experiments}

Barotropic case $\left(\rho_{1} \approx \rho_{0}\right)$. Before the beginning of each experiment, the tank was filled with distilled water to the level of the upper edge of the ring source. The Mariotte bottle was filled with water of the same density, but tinted with thymol dye. Then, the rotation of the platform with an angular velocity $\Omega$, varying from 0.6 to $1.25 \mathrm{rad} / \mathrm{s}$ was started. When the water in the tank was spinned up to a state close to solid-state rotation, video cameras and a ring source of constant fluid flow rate were switched on. The moment of inclusion of the latter was taken as the beginning of the experiment.
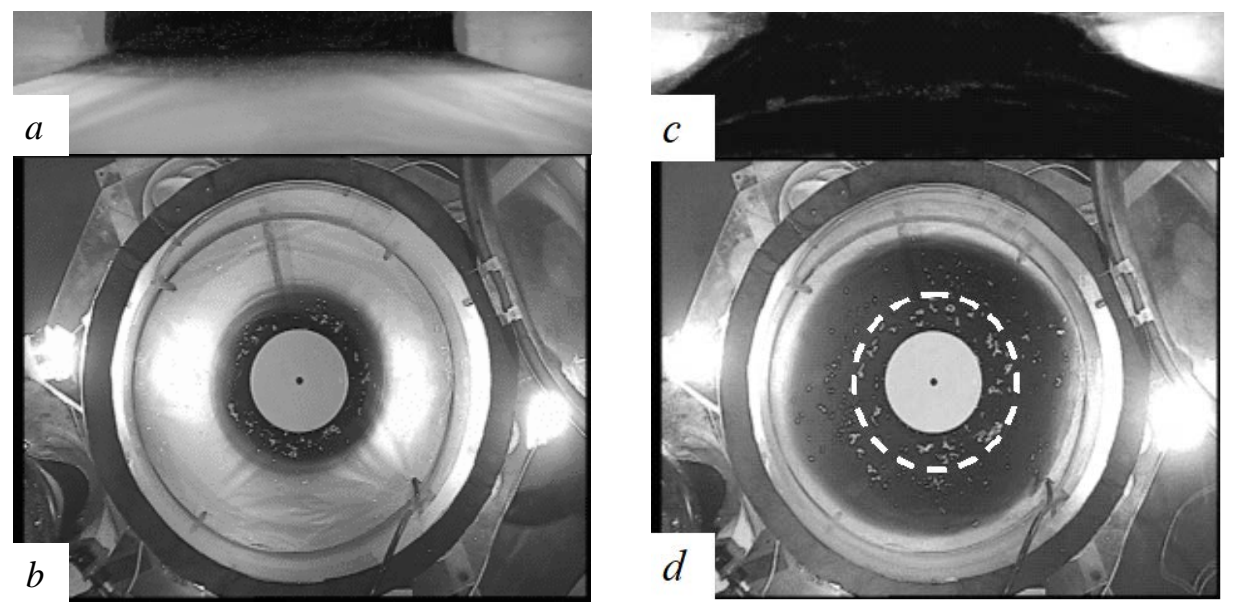

Fig. 2. Barotropic frontal current above the cone surface formed by the constant source $\left(Q=8 \mathrm{~cm}^{3} / \mathrm{s}\right.$, $\left.f=1.25 \mathrm{~s}^{-1}\right): a, b$ - the initial stage of the experiment when the current inviscid core is being formed: $a$ - side view; $b$ - view from above; $c, d$ - the late stage of the experiment when the inviscid core has already reached the quasi-stationary state (white dotted line denotes the external frontal boundary of the current inviscid core), and the bottom Ekman layer has spread far along the cone surface: $c$ - side view; $d$ - view from above

The tinted fluid coming from the source to the surface of the cone initially formed an anticyclonic axisymmetric barotropic coastal current that expanded radially (Fig. 2, $a, 2, b$ ). After a while, the width of the layer of the tinted fluid and the associated coastal current practically ceased to grow, despite the continued inflow 
of the fluid from the source. The stabilization of the tinted fluid front position was due to the formation of a flow in the viscous bottom Ekman layer at a rate of $Q_{e} \approx$ $\approx Q$ [8], propagating down the slope and leaving the frontal zone of the barotropic layer of the tinted fluid far beyond (Fig. 2, $c, 2, d$ ). At the same time, the coastal current also reached a steady state, and its centerline (the azimuthal velocity maximum) was located on the outer boundary of the barotropic layer of the tinted fluid.

For each experiment, the width of the ring axisymmetric front current, as well as the width of the bottom Ekman layer ring, were measured according to the top and lateral video survey data at successive times. According to visual observations, the first parameter reached a quasi-stationary value quickly, and the second one continued to grow. By the displacement of paper pellets, the azimuthal velocity of the barotropic current in the area of its front (centerline) was also measured, and the creep speed of the tinted water of the source in the bottom Ekman layer was calculated. It should be noted that the fluid in the bottom layer also had an azimuthal, anticyclonic directional velocity component, which fully corresponds to the theoretical concepts of the dynamics of the bottom Ekman layer. In this case, the thickness of the viscous bottom flow at the outer boundary of the barotropic layer of the tinted fluid should be proportional to the Ekman scale.

In the experiments of the first series, the purpose to determine the regularities of the bottom Ekman layer propagation down the surface of a smooth cone, depending on the dimensional and dimensionless parameters of the problem was set. The depth of the basin outside the cone and the radius of the cone are assumed to be much larger than the height of the source and its radius, respectively, and that these two parameters have no effect on the formation of the front current and the bottom Ekman layer on the cone. In this case, the defining dimensional parameters of the problem are the following: $Q\left(\mathrm{~cm}^{3} / \mathrm{s}\right)$ is the source flow rate, $v\left(\mathrm{~cm}^{3} / \mathrm{s}\right)$ is the kinematic viscosity of the water, $R_{0}(\mathrm{~cm})$ is the source radius, $H_{0}$ is the height of the water layer at the external source boundary, $f=2 \Omega\left(\mathrm{s}^{-1}\right)$ is the Coriolis parameter. The dimensionless parameter $\operatorname{tg} \alpha$ - the slope of the bottom of the basin to the horizontal, also plays an important role.

Of the five above dimensional parameters, two have an independent dimension. According to the $\Pi$-theorem, three dimensionless parameters can be composed. These completely determine the kinematic characteristics of the barotropic front current and the current in the bottom Ekman layer: $\mathrm{Re}=\mathrm{Q} / \mathrm{vH}_{0}$ is the Reynolds number; $\mathrm{Ek}=v / f \mathrm{H}_{0}{ }^{2}$ is the Ekman number; $\theta=R_{0} / H_{0}$ is the source form factor. To these three dimensionless parameters, the fourth one is added: $\operatorname{tg} \alpha$ is the bottom slope. In the experiments, the values of $Q$ and $f$ parameters change, so the $\theta$ and $\operatorname{tg} \alpha$ dimensionless parameters are constants of the experiment.

As mentioned above, the structure of the current field produced by a constant flow rate source quickly reaches a quasistationary state. At the same time, in some zone near the source there is an inviscid core of the barotropic current bounded by the front of the tinted fluid from the source. Outside the source, in the surrounding fluid, the quasistationary state of an inviscid core is maintained by the inflow from a water source with a small angular momentum, and also by bottom friction, which is the cause of the spin-up (untwisting) of the overlying fluid. Some inviscid core radius increase with time (Fig. $3, a$ ) is due to a general increase in the water mass $\mathrm{n}$ the basin. 
In a quasistationary state, the flow rate of fluid coming from the source is compensated by its outflow and downward slope in the viscous (Ekman) bottom layer, whose width $L_{e}$, measured from the outer boundary of the coastal current, increases in proportion to $t^{1 / 2}$ (Fig. $3, b$ ). This means that the thickness of the bottom Ekman layer does not change with the distance from the front of the coastal current, and the velocity of the flow behind the front changes (decreases) inversely with this distance.
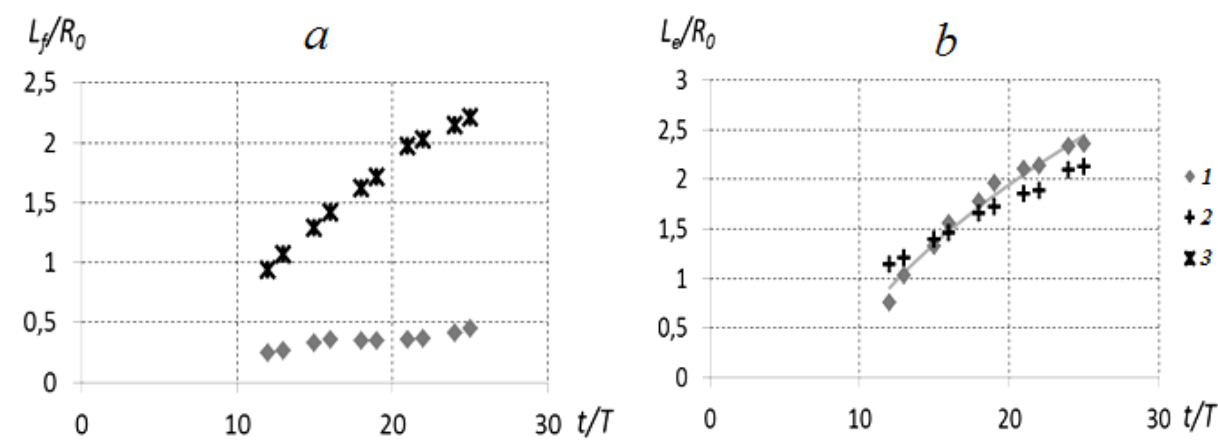

Fig. 3. Dependences: $L_{f} / R_{0}$ (a) and $L_{e} / R_{0}$ (b) on the dimensionless time $t / T$, where $T=2 \pi / f$ is the period of the platform rotation; $R_{0}$ is the source radius. $Q=8 \mathrm{~cm}^{3} / \mathrm{s}, f=1.25 \mathrm{~s}^{-1} .1$ is the barotropic case $\left(g^{\prime}=0\right)$; 2 is the baroclinic case $\left(g^{\prime}=0.28\right)$; 3 is the baroclinic case $\left(g^{\prime}=1.12\right)$. The curve is the approximation of the barotropic bottom Ekman layer radius by the function proportional to the square root of time. The dimensionless time $t / T$ is represented in the laboratory day unit (lab. day)

In this case, the integral flow rate of the fluid in this layer is

$$
Q_{E}=Q=2 \pi\left(R_{0}+L_{f}\right) V_{f} H_{e},
$$

where $L_{f}$ is the width of the inviscid core of the flow, i. e. the radial distance from the outer boundary of the cylindrical source to the point of the tinted fluid that coincides with the centerline. The azimuthal current velocity equals $U_{f}$, and $V_{f}$ is the radial component of the current velocity in the bottom Ekman layer of $H_{e}$ thickness at $L_{f}$ distance from the source.

From the viewpoint of physics, the velocity of radial propagation of the fluid $V_{f}=d L_{e} / d t$ in the Ekman layer at the external boundary of the inviscid core of the frontal downwelling current must be proportional to the azimuthal current velocity $U_{f}$ and the cosine of the bottom inclination angle. At the same time, as already indicated, the real thickness of the near-bottom viscous boundary layer $H_{e}$ should be proportional to the Ekman scale of thickness $h_{e}=(v / f \cos \alpha)^{1 / 2}$ taking into account the bottom slope. In other words, taking into account the constancy of the bottom inclination angle in the experiment,

$$
\begin{gathered}
V_{f}=C_{1} U_{f}, \\
H_{e}=C_{2} h_{e} .
\end{gathered}
$$

Here $C_{1}$ and $C_{2}$ - are constants that can be functions of the Reynolds and Ekman numbers. $C_{1}$ constant can be determined from the experiment by measuring $U_{f}$ and $V_{f}$ simultaneously. Since the Ekman boundary layer on the inclined bottom is very 
thin (not more than $1 \mathrm{~cm}$ ), its thickness is difficult to measure. But using the expressions (1), (2) and (3), $C_{2}$ constant can be expressed in terms of other parameters of the problem:

$$
C_{2}=Q / 2 \pi\left(R_{0}+L_{f}\right) C_{1} U_{f} h_{e} .
$$

Applying the potential vortex conservation law, it can be shown that the velocity in the barotropic coastal current centerline is determined by the following scale

$$
U_{f}^{*}=-\left(H_{0} / H_{f}\right) f L_{f}
$$

One of the tasks of the experimental data processing was to check the dependence

$$
U_{f}=C_{3} \cdot U_{f}^{*}
$$

and to determine $C_{3}$ coefficient value. This coefficient can also be a function of dimensionless parameters - the Reynolds and the Ekman number (see above).

Substituting in the formula (4) the expressions (5) and (6) for the corresponding scales, the following is obtained

$$
Q=2 \pi\left(R_{0}+L_{f}\right) C\left(H_{0} / H_{f}\right) f L_{f} h_{e}
$$

where $C=C_{1} C_{2} C_{3}$.

Provided that $R_{0}>>L_{f}$ and $H_{0} \approx H_{f}$, the expression (7) is simplified and converted to the following form

$$
Q=2 \pi R_{0} C f L_{f}^{*} h_{e} .
$$

Here $L_{f}^{*}$ is the scale width of the barotropic inviscid core of the frontal current under the conditions indicated above. From the formula (8) it is possible to express this scale in the following form

$$
L_{f}^{*}=Q / 2 \pi R_{0} C f h_{e} .
$$

In total, five experiments with a barotropic coastal current were carried out. The dimensional parameters $Q$ and $f$ there assumed the following values: 4, 6, $10 \mathrm{~cm}^{3} / \mathrm{s}$ and $0.625 ; 1.25 ; 2.51 / \mathrm{s}$, respectively. The dimensionless parameters - of the Reynolds and Ekman numbers - varied in the following ranges: $200<\mathrm{Re}<$ $<$ 500; $0.001<$ Ek $<0.004$.

From the results of laboratory experiments, the following values of the constants introduced above were obtained: $C_{1}=0.06 \pm 0.005 ; C_{2}=11.3 \pm 1.7 ; C_{3}=$ $=0.82 \pm 0.08 ; C=0.5 \pm 0.12$. The essential dependence of these constants on the dimensionless parameters of the problem - the Reynolds and Ekman numbers was not revealed.

Baroclinic case $\left(\boldsymbol{\rho}_{\mathbf{1}}<\boldsymbol{\rho}_{\mathbf{0}}\right)$. In this case, another defining dimension parameter of the problem appears: the reduced free fall acceleration $g^{\prime}=g \Delta \rho / \rho_{0}$. Here $g$ is the free fall acceleration; $\Delta \rho=\rho_{0}-\rho_{1}=\beta\left(S_{0}-S_{1}\right)$, where $\beta$ is the haline contraction coefficient; $S_{0}$ and $S_{1}$ is the water salinity in in the source and in the tank. Applying $g^{\prime}$ it is possible to construct another important dimensional parameter of the problem - the Rossby baroclinic deformation radius $R_{d}=g^{\prime} H_{0} / f$. Looking ahead, it should be noted that the dimensionless parameter $R_{d} / L_{f}^{*}$ determines the condition for water from the source to reach the bottom Ekman layer.

PHYSICAL OCEANOGRAPHY VOL. 25 ISS. 3 (2018) 


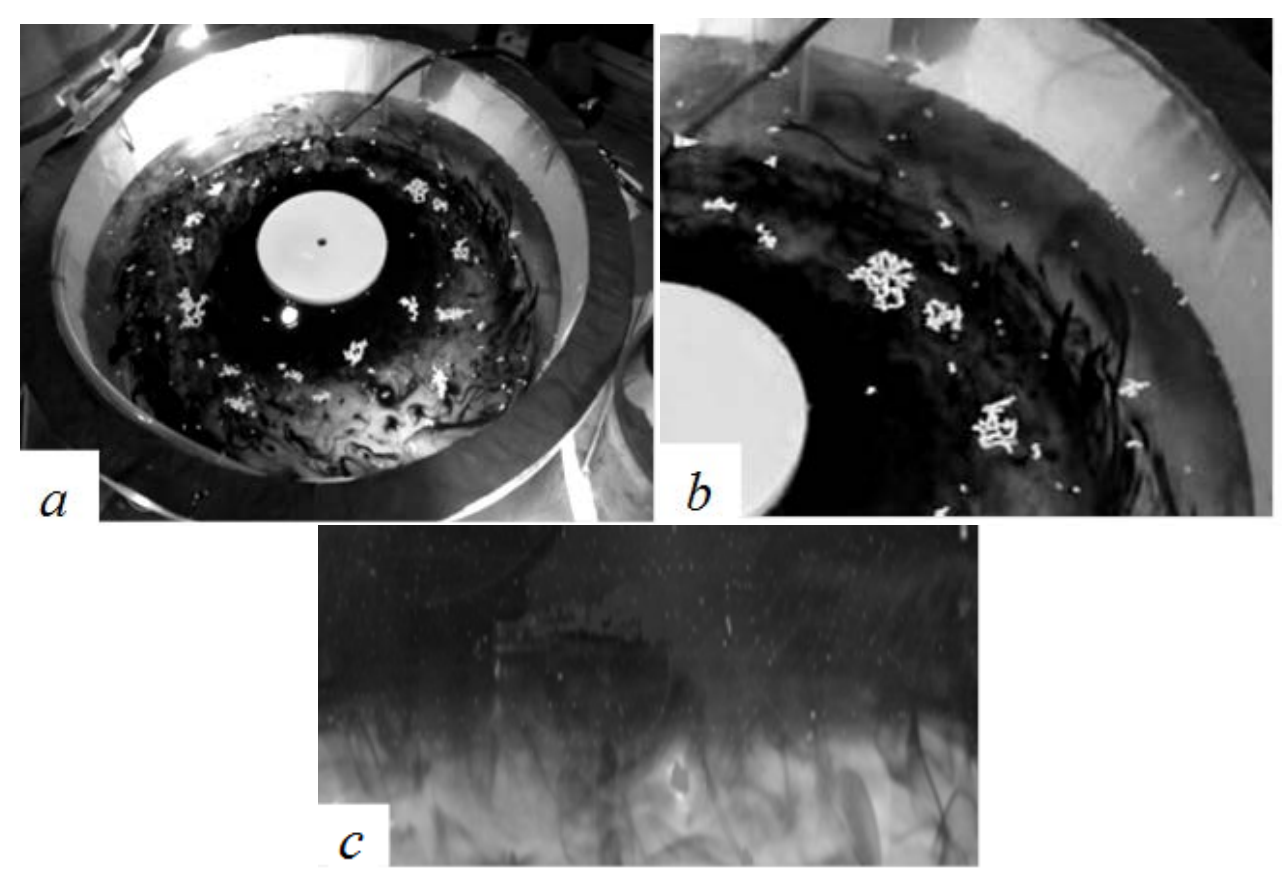

Fig. 4. Current structure at insignificant difference between the water density in the source and the one in the tank $\left(Q=8 \mathrm{~cm}^{3} / \mathrm{s}, f=1.25 \mathrm{~s}^{-1}, g^{\prime}=0.28 \mathrm{~cm} / \mathrm{s}^{2}\right): a$ - side view, $b$ - view from above; $c$ three-dimensional convective structures

In experiments, when the density of the waters of the frontal current was less than the density of the fluid in the tank, two different scenarios for the frontal current evolution were realized. In the first scenario, typical for a relatively small difference in the water density in the source from the one in the basin, the current front slightly inclined to the vertical reached the surface of the cone and the bottom Ekman layer was formed, in which more light water descended down the slope. The speed of sinking was somewhat smaller as compared to the barotropic case (Fig. 3, b). In course of time, the bottom Ekman layer experienced convective instability: it formed roller structures, stretched in the azimuth direction. They then disintegrated into three-dimensional eddy structures in which the tinted water of the bottom Ekman layer rose slowly up to the free surface of the fluid. In Fig. 4 the structure of the current with a slight difference in the water density in the source from one in the tank is shown. At the same time, Fig. 4, a presents a general view of the current at an angle of about $45^{\circ}$ : the inviscid core of the coastal current is seen as a dark ring of tinted fluid around the source, and on its periphery eddy elements of convection generated by the bottom Ekman layer convective instability are noticeable. Fig. 4, $b$ shows the tank segment with a front current and eddy elements of convection, and in Fig. 4, the zoomed picture shows the three-dimensional elements of convection, extending from the bottom to the surface of the water.

The quasilaminar nature of convection was due to the relatively weak supercriticality of the Rayleigh number for the bottom Ekman layer: because of the effect of rotation with the Taylor number growth, the critical Rayleigh number also increases [9]. 

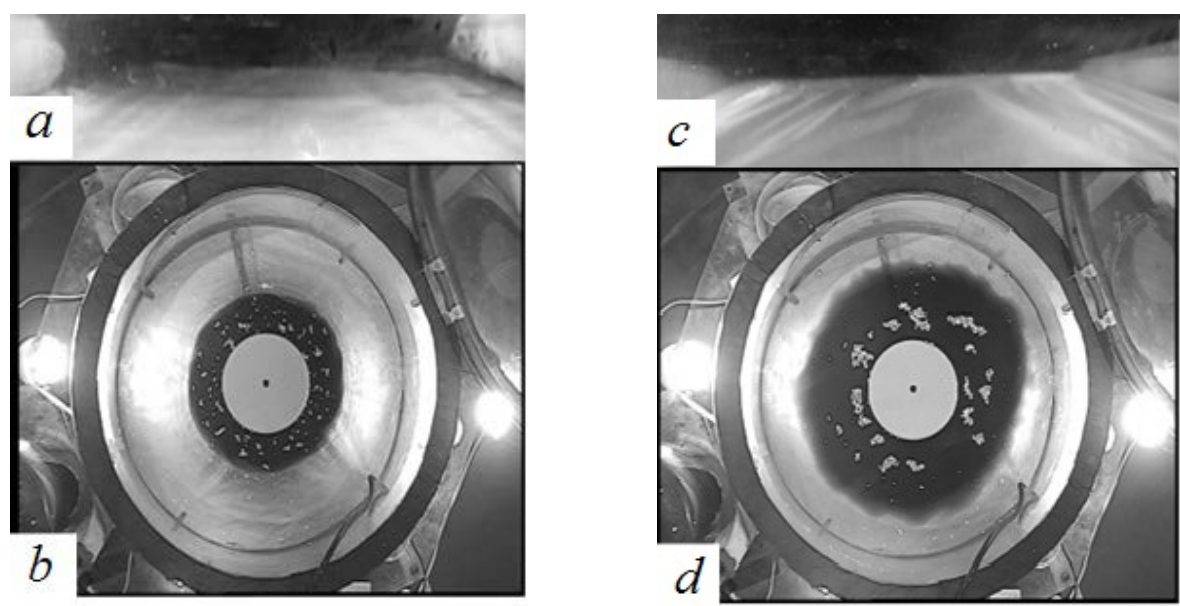

Fig. 5. Structure of the well pronounced baroclinic current $\left(Q=8 \mathrm{~cm}^{3} / \mathrm{s}, f=1.25 \mathrm{~s}^{-1}, g^{\prime}=1.12 \mathrm{~cm} / \mathrm{s}^{2}\right)$ : $a, b$ - the initial stages of the experiment: $a$ - side view, $b$ - view from above; $c, d$ - the late stages of the experiment: $c$ - side view, $d$ - view from above. No entering of the frontal current water to the bottom Ekman layer

In the second scenario, typical one in the tank, the inclined current front did not reach the surface of the cone. In this case, the tinted and lighter water from the source did not fall into the bottom Ekman layer (Fig. 5, $a, 5, b$ ). With the source operating, the mass of fluid enclosed within the coastal current area grew continuously and the position of its front did not stabilize: its radial propagation took place (Fig. 5, $c, 5, d$ ). It is known that, above a horizontal flat bottom, the baroclinic current, as a rule, loses stability, meanders and falls into eddy structures. However, over the inclined bottom, the current stability is substantially increased due to the stabilizing role of topography [10].

In the context of this article, it is necessary to discuss the conditions for the transition from barotropic current regime in the bottom Ekman layer to the baroclinic one, and actually, - the convective instability of the bottom Ekman layer. Since the thickness of the water layer in the tank $H(\sim 10 \mathrm{~cm})>>H_{e}$, it can be assumed that the presence of convective instability does not depend on $H$, but is determined by the scale of the thickness of the bottom Ekman layer. It is as if the convective instability appears when the value of the Rayleigh number becomes critical for the boundary layer

$$
\mathrm{Ra}=g^{\prime} H_{e}^{3} / k_{s} v .
$$

Here $k_{s}$ is the salt diffusion coefficient, and $v$ is the kinematic water viscosity. From laboratory experiments it follows that the convective instability of the bottom Ekman layer occurs when $\left(g^{\prime}\right) * \geq 0.07 \mathrm{~cm} / \mathrm{s}^{2}, f=1.25 \mathrm{~s}^{-1}$ and $H_{e} \approx 1.0 \mathrm{~cm}$. Taking $k_{s}=1.4 \cdot 10^{-5} \mathrm{~cm}^{2} / \mathrm{s}$ and $v=10^{-2} \mathrm{~cm}^{2} / \mathrm{s}$ and substituting the values of the parameters in the expression (10), $\mathrm{Ra}_{\text {crit }} \approx 5 \cdot 10^{5}$ is obtained. This value is approximately four orders of magnitude greater than for a cold thermal boundary layer in water near the water-air interface in the non-rotating fluid $\left(\mathrm{Ra}_{\text {crit }}=64\right.$ [11]). As already noted, in a rotating fluid the development of free convection begins at large values of the Rayleigh number (the rotation "stabilizes" free convection), and the critical Rayleigh number is an increasing function of the Taylor number Ta $=f^{2} \mathrm{H}_{e}{ }^{4} / v^{2}$. In the case under consideration $\mathrm{Ta} \approx(1-2) \cdot 10^{4}$. Starting from the diagrams of the convec- 
tive instability of boundary layers in the rotating fluid, given in [9], for such values of the Taylor number, the critical value of the Rayleigh number should be about one and a half two orders of magnitude less than the estimate given above. The reason for this difference is the incomplete laminar bottom Ekman layer and the presence of a shear flow in it. Observations show that it is also subject to nonlinear wave motions, which can increase the diffusion of salt and momentum in comparison with pure molecular metabolism. Evidence that this layer is not laminar is also its relatively large thickness: it is an order of magnitude greater than the Ekman scale calculated using the molecular viscosity of water. It can be said that this layer is subject to wave-eddy perturbations. In this case, instead of the molecular exchange coefficients in the expression for the critical Rayleigh number (10), the effective salt and momentum exchange coefficients should be used. In addition, the actual value of $g^{\prime}$ may be less than the salinity calculated between the water in the source and in the tank due to partial mixing of the interacting waters. All this can lower the value of the critical Rayleigh number by more than an order of magnitude.

This issue obviously needs further laboratory and theoretical study. This present problem is most likely not relevant to the in situ conditions, because in the sea the bottom Ekman layer is turbulent and extended along the vertical, and the Earth's rotation effect on the development of the bottom Ekman layer instability is insignificant.

As already noted above, with a large difference in the salinity (density) of water in the tank and in the source, a second scenario is realized: the coastal downwelling current wedges to the water surface in the tank, becomes thin and does not touch the bottom anywhere. In this case, the inviscid core of the current grows longer, reaching a greater width than in the barotropic case (Fig. 3, a). It can be shown that the condition for the beginning of the second scenario is achieved if the value of the baroclinic deformation radius exceeds the value of the scale of the width $L_{f}^{*}$ of the inviscid core of the barotropic coastal current defined by the expression (9). In this case, the condition is as follows

$$
L_{f}^{*}<R_{d}=\left(g^{\prime} H_{0}\right)^{1 / 2} / f \text {. }
$$

From this condition (11), the threshold value $\left.g^{\prime}\right)^{* *}$ can be defined. At this value the baroclinicity begins to exert a decisive effect on the current regime:

$$
\left(g^{\prime}\right) *\left(L_{f}^{*} f\right)^{2} / H_{0} \text {. }
$$

Substituting in the expression (12) the expression (9) for $L_{f}^{*}$ and $h_{e}=(v / f)^{1 / 2}$, the following expression is obtained

$$
\left(g^{\prime}\right)^{* *}>\left(Q f^{1 / 2} /\left(2 \pi C R_{0} H_{0}^{1 / 2} v^{1 / 2}\right)\right)^{2}=C_{4}\left(Q f^{1 / 2} /\left(2 \pi R_{0} H_{0}{ }^{1 / 2} v^{1 / 2}\right)\right)^{2} .
$$

From laboratory experiments it follows that in the baroclinic case the transition from convectively stable bottom Ekman layer to convectively unstable occurs for $\left(g^{\prime}\right)^{*}=0.07 \mathrm{~cm} / \mathrm{s}^{2}$ when $f=1.25 \mathrm{~s}^{-1}$ and $H_{e} \approx 1.0 \mathrm{~cm}$. At that transition from the first scenario to the second one occurs when $\left(g^{\prime}\right)^{* *}=0.84 \mathrm{~cm} / \mathrm{s}^{2}$.

Analysis of the experimental data showed that $C_{4}=0.4 \pm 0.05$. A considerable dependence of this constant on the dimensionless parameters of the experiment is not also found.

\section{REFERENCES}

1. Munk, W.H., 1966. Abyssal Recipes. Deep Sea Research and Oceanographic Abstracts, [e-journal] 13(4), pp. 707-730. https://doi.org/10.1016/0011-7471(66)90602-4 
2. Zatsepin, A.G., Golenko, N.N., Korzh, A.O., Kremenetskii, V.V., Paka, V.T., Poyarkov, S.G. and Stunzhas, P.A., 2007. Influence of the Dynamics of Currents on the Hydrophysical Structure of the Waters and the Vertical Exchange in the Active Layer of the Black Sea. Oceanology, [e-journal] 47(3), pp. 301-312. https://doi.org/10.1134/S0001437007030022

3. Zhurbas, V.M., Oh, I.S. and Park, T., 2006. Role of the Beta-Effect in the Decay of the Alongshore Baroclinic Jet Associated with Transient Coastal Upwelling and Downwelling: Numerical Experiments. Oceanology, [e-journal] 46(2), pp. 170-177. https://doi.org/10.1134/S0001437006020032

4. Schaeffer, A., Roughan, M. and Wood, J.E., 2014. Observed Bottom Boundary Layer Transport and Uplift on the Continental Shelf Adjacent to a Western Boundary Current. Journal of Geophysical Research, [e-journal] 119(8). doi:10.1002/2013JC009735

5. Moum, J.N., Perlin, A., Klymak, J.M., Levine, M.D., Boyd, T. and Kosro, P.M., 2004 Convectively Driven Mixing in the Bottom Boundary Layer. J. Phys. Oceanogr., [e-journal] 34(10), pp. 2189-2202. https://doi.org/10.1175/1520-0485(2004)034<2189:CDMITB>2.0.CO;2

6. Ostrovskii, A.G. and Zatsepin, A.G., 2016. Intense Ventilation of the Black Sea Pycnocline due to Vertical Turbulent Exchange in the Rim Current Area. Deep-Sea Res. I, [e-journal] 116, pp. 1-13. https://doi.org/10.1016/j.dsr.2016.07.011

7. Elkin, D.N., Zatsepin, A.G., Podymov, O.I. and Ostrovskii, A.G., 2017. Sinking of Less Dense Water in the Bottom Ekman Layer Formed by a Coastal Downwelling Current over a Sloping Bottom. Oceanology, [e-journal] 57(4), pp. 478-484. https://doi.org/10.1134/S0001437017040051

8. Zatsepin, A.G. and Semenov, A.V., 1997. Barotropnye Vikhri-Intruzii nad Ploskim Dnom vo Vrashchayushcheysya Zhidkosti [Barotropic Vortex-Intrusions over a Flat Bottom in a Rotating Fluid]. In: Intruzionnye i Plotnostnye Techeniya (Pamyati V. N. Anuchina) [Intrusive and Dense Currents (memory of Anuchin V.N.)]. Kaliningrad: Kaliningrad State Technical University, pp. 55-69 (in Russian).

9. Boubnov, B.M. and Golitsyn, G.S., 1995. Convection in Rotating Fluids. Dordrecht: Kluwer Academic Publishers, $224 \mathrm{p}$.

10. Didkovskii, V.L., Semenov, A.V. and Zatsepin, A.G., 2000. Mesoscale Currents upon the Smooth Sloping Bottom and in the Presence of Ridges and Canyons. In: UNESCO, 2000. Oceanic Fronts and Related Phenomena: Konstantin Fedorov International Memorial Symposium, Pushkin, Saint Petersburg, 18-22 May 1998: Proceedings. IOC Workshop report N 159. UNESCO, pp. 89-94.

11. Ginsburg, A.I. and Fedorov, K.N., 1978. O Kriticheskom Pogranichnom Chisle Releya pri Okhlazhdenii Vody cherez Svobodnuyu Poverkhnost' [On the Critical Value of the Boundary Rayleigh Number Assotiated with the Cooling of Water through Free Surface]. Izvestiya of the Academy of Sciences of the USSR. Atmospheric and Oceanic Physics, 14(4), pp. 433-436 (in Russian).

About the authors:

Dmitriy N. Elkin - Junior Research Associate, Shirshov Institute of Oceanology, Russian Academy of Science (36, Nahimovskiy pr., Moscow, 117997, Russia)

Andrey G. Zatsepin - Head of the Laboratory, Russian Academy of Science (36, Nahimovskiy pr., Moscow, 117997, Russia), Dr.Sci. (Phys.-Math.), ORCID ID: 0000-0002-5527-5234, zatsepin@ocean.ru

Contribution of the co-authors:

Dmitriy N. Elkin - the laboratory installation modernization, carrying out experimental research, data processing, constructing dependencies, writing the original text of the article, preparing the article figures

Andrey G. Zatsepin - task setting of the study, participation in the data analysis, in the formulation of the study results and conclusions, in writing the article and its revision based on the reviewer comments

All authors have read and approved the final manuscript.

The authors declare that they have no conflict of interest.

PHYSICAL OCEANOGRAPHY VOL. 25 ISS. 3 (2018) 